\title{
A Catheter-Related Bloodstream Infection Caused by the Yeast Kodamaea ohmeri
}

\author{
Ding $\mathrm{CH}^{\mathrm{a}}$, Tzar $\mathrm{MN}^{\mathrm{b}}$, Biswas $\mathrm{S}^{\mathrm{c}}$, Muttaqillah $\mathrm{NAS}^{\mathrm{d}}$, Wahab $\mathrm{AA}^{\mathrm{e}}$ \\ a-e Department of Medical Microbiology and Immunology, Level 16, Pre-Clinical Building, UKM Medical \\ Centre, Jalan Yaakob Latif, 56000 Cheras, Kuala Lumpur, Malaysia
}

\begin{abstract}
Catheter-related bloodstream infections caused by Kodamaea ohmeri are generally not considered due to the relative scarcity of reported cases. This is a case of an 85-year-old man with poorly controlled diabetes mellitus who was initially admitted to our hospital for diabetic ketoacidosis. An internal jugular catheter was inserted as part of the initial management. A week later the patient developed a temperature spike and a yeast identified as Kodamaea ohmeri by ID $32 \mathrm{C}$ (bioMérieux, France) was isolated from both his central and peripheral blood cultures. The catheter was removed and the patient was treated with fluconazole despite the organism's relatively high minimum inhibitory concentration $(2 \mu \mathrm{g} / \mathrm{mL})$ to this antifungal. The fungemia resolved following a 2 -weeks course of fluconazole.
\end{abstract}

KEYWORDS: Kodamaea ohmeri, catheter, fungemia, fluconazole

\section{INTRODUCTION}

The landscape of invasive mycoses in humans has been altered significantly in the past decade, with more and more unusual fungal agents being implicated. When the yeast Kodamaea ohmeri was first reported in 1998, it was considered a contaminant but it is now deemed to be an important human pathogen that has been associated with fungemia, funguria, endocarditis, and peritonitis. $^{1}$ Kodamaea ohmeri is an unusual organism because it can be mistaken for a more commonly isolated yeast (e.g. Candida) and because of its reduced susceptibility to a commonly used antifungal agent such as fluconazole. ${ }^{1}$ Although its accurate mycological diagnosis is relatively simple, it is likely that Kodamaea ohmeri may be erroneously identified in many hospital laboratories. $^{2}$

Corresponding author

Dr. Asrul Abdul Wahab

Department of Medical Microbiology and

Immunology, UKM Medical Centre,

56000 Cheras, Kuala Lumpur

Tel: 03-91459530

Email: asrulwahab@hotmail.com

\section{CASE REPORT}

An 85-year-old Malay man with hypertension, diabetes mellitus and chronic renal disease presented to the Emergency Department of UKM Medical Centre with a 2-day history of fever, nonproductive cough and shortness of breath. He had been wheelchair-bound for several years following a below-knee amputation for diabetic complications.

On initial clinical examination, the patient had a temperature of $37.8^{\circ} \mathrm{C}$, a heart rate of 103 beats/ min, a respiratory rate of 45 breaths/min and his blood pressure was 113/76 mmHg. Respiratory system examination revealed decreased air entry at the bases of both lungs with audible crepitations. His bedside urine dipstick revealed the presence of ketones $(2+)$. The glucometer reading was "high". The arterial blood gas analysis (on high flow $\mathrm{O}_{2}$ mask) revealed a $\mathrm{pH}$ of $7.12, \mathrm{PaCO}_{2}$ of $25 \mathrm{mmHg}$, $\mathrm{PaO}_{2}$ of $76 \mathrm{mmHg}$, and $\mathrm{HCO}_{3}{ }^{-}$of $9.5 \mathrm{mmol} / \mathrm{L}$. A provisional diagnosis of severe metabolic acidosis secondary to diabetic ketoacidosis was made. The patient developed respiratory distress with Kussmaul's breathing and had to be mechanically ventilated in the intensive care unit. A central venous catheter was also placed in his internal jugular vein. 
Initial investigations revealed a total white blood cell count of $11.7 \times 10^{9} / \mathrm{L}$, a hemoglobin level of 9.2 $\mathrm{g} / \mathrm{dL}$, a platelet count of $201 \times 10^{9} / \mathrm{L}$, a urea level of $15.3 \mathrm{mmol} / \mathrm{L}$, a serum creatinine of $200 \mu \mathrm{mol} / \mathrm{L}$, and a C-reative protein level of $3.29 \mathrm{~g} / \mathrm{dL}$. The chest X-ray showed bilateral lower zone consolidation. A diagnosis of bacterial pneumonia was made and specimens (blood and tracheal aspirate) were sent for microbiological diagnosis. Intravenous ceftriaxone $1 \mathrm{~g}$ daily and oral azithromycin $500 \mathrm{mg}$ daily were started empirically. The first blood culture yielded no growth but Staphylococcus aureus was isolated from the tracheal aspirate.

On day 7 of admission a temperature of $39^{\circ} \mathrm{C}$ was recorded after an afebrile period of 3 days. Concurrent blood specimens were taken from the central venous catheter and from a peripheral (arm) vein for culture. Both the central and peripheral blood specimens in BD BACTEC ${ }^{\top M}$ Plus Aerobic/F vials (Becton, Dickinson and Company, USA) were positive for yeasts, with the former being positive first. The differential time to positivity exceeded 2 hours. An $800 \mathrm{mg}$ stat dose of fluconazole was given intravenously as empirical treatment, followed by daily fluconazole doses of $\mathbf{2 0 0} \mathbf{m g}$. The central line was also removed.

When the yeasts were cultured on chromogenic agar, small pink colonies appeared after an overnight incubation at $35^{\circ} \mathrm{C}$. At the same time, culture on cornmeal agar was also done and after 24 hours of incubation at $25^{\circ} \mathrm{C}$ the microscopic morphology was suggestive of Candida tropicalis (Figure 1). However, if indeed the yeast was Candida tropicalis, blue colored colonies should have been seen on chromogenic agar. Thus, due to this discrepancy, carbohydrate assimilation testing using ID 32 C (bioMérieux, France) was performed and the yeast was identified as Kodamaea ohmeri (numerical profile 5167150015; $99.8 \%$ identification). Also, following extended incubation, the colonies started to change colour to blue, as shown in the 5 -day old culture in Figure 2 . Antifungal susceptibility testing was performed with the broth microdilution method (Sensititre ${ }^{\circledR}$ YeastOne ${ }^{\circledR}$ Y010) (TREK Diagnostic Systems, USA). The minimum inhibitory concentration (MIC) values were read at 24 hours and are shown in Table 1.

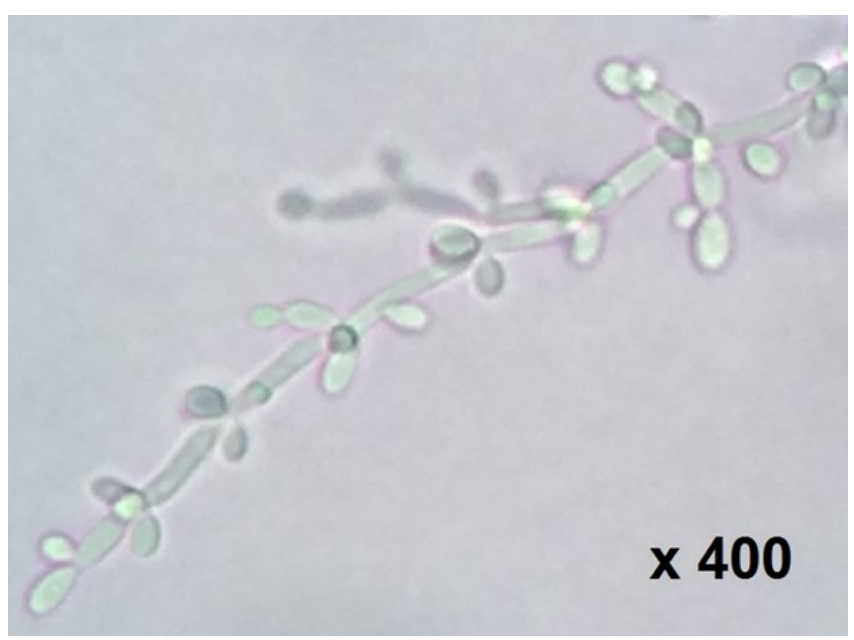

Figure 1: Microscopic morphology of Kodamaea ohmeri on cornmeal agar showing blastoconidia being produced singly or in small groups along the sides of pseudohyphae.

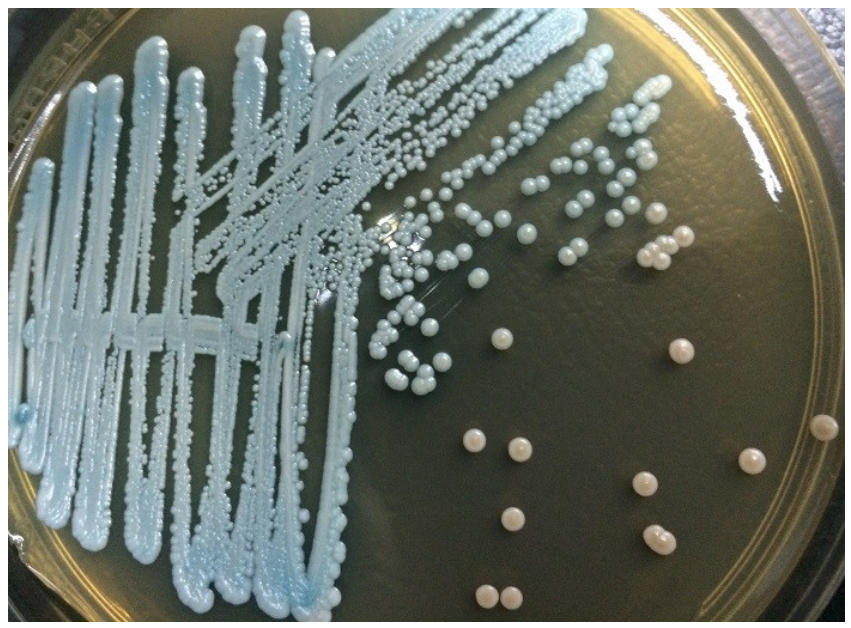

Figure 2: Kodamaea ohmeri colonies on chromogenic agar after 5 days of incubation.

Table 1: Antifungal MIC results for Kodamaea ohmeri

\begin{tabular}{cc}
\hline Antifungal agent & MIC $(\mu \mathrm{g} / \mathrm{mL})$ \\
\hline Amphotericin B & 0.25 \\
Anidulafungin & 0.25 \\
Micafungin & 0.06 \\
Caspofungin & 0.06 \\
Posaconazole & 0.015 \\
Voriconazole & 0.015 \\
Itraconazole & 0.06 \\
Fluconazole & 2 \\
\hline
\end{tabular}

Despite the elevated fluconazole MIC (compared to those of other antifungals), fluconazole was administered for a total duration of 14 days. The fungemia cleared following the 2-week course of fluconazole, as evidenced by the absence of growth in a repeat blood culture taken at this time and the patient was discharged home. 


\section{DISCUSSION}

The fungal pathogens most frequently reported as etiological agents of invasive mycoses are Candida spp., Aspergillus spp. and the Zygomycetes. ${ }^{3}$ Kodamaea ohmeri is a fungal pathogen that has been rarely reported in the medical literature. ${ }^{4}$ This ascomycetous environmental yeast was formerly classified in the genus Pichia and was previously known as Pichia ohmeri and among the five species in the Kodamaea genus, only Kodamaea ohmeri is considered a human pathogen. ${ }^{2}$

Our patient's poorly controlled diabetes mellitus is likely to have predisposed him to the Kodamaea ohmeri infection. The usage of indwelling medical devices such as intravascular catheters is also recognized as a predisposing factor for opportunistic yeast infections, and our patient had an indwelling central venous catheter for a week before the fungemia was detected. This specific association between catheter usage and Kodamaea ohmeri fungemia is consistent with the published literature that up to $70 \%$ of cases are catheterrelated.

The accurate laboratory diagnosis of Kodamaea ohmeri can be challenging in the absence of a high index of suspicion. An important clue is the inconsistency between early (24-hour) cornmeal and chromogenic agar findings. The growth of small pink colored colonies on chromogenic agar would suggest either Candida parapsilosis or Candida glabrata, rather than Candida tropicalis. $^{5}$ However, an extended incubation on chromogenic agar will result in some colonies undergoing a color transition from pink to blue. This color-changing tendency of Kodamaea ohmeri has also been reported by other authors. $^{6}$

The significance of this yeast lies in its potential resistance to fluconazole, an antifungal agent favored for its flexible route of administration, low cost and good side-effect profile. Shang et al (2010) reported that the fluconazole MIC for Kodamaea ohmeri ranged from 0.125 to $64 \mu \mathrm{g} / \mathrm{mL}$, but the Clinical and Laboratory Standards Institute (CLSI) does not provide specific breakpoints to interpret antifungal MICs for non-Candida yeasts. ${ }^{7}$ Our Kodamaea ohmeri isolate had a fluconazole MIC of $2 \mu \mathrm{g} / \mathrm{mL}$, which is the highest MIC denoting susceptibility if interpreted according to the CLSI breakpoints available for Candida spp.

Thus, it has been suggested by Yang et al (2009) that Kodamaea ohmeri infections be treated with amphotericin B while Shang et al (2010) suggested one of the newer triazoles (e.g. voriconazole), or an echinocandin. We were therefore fortunate that we succeeded in clearing our patient's fungemia with fluconazole although the removal of his intravascular catheter is likely to have also contributed to the clearance as well.

\section{CONCLUSION}

In conclusion, when faced with the clinical diagnosis of a fungal catheter-related bloodstream infection, one should consider Kodamaea ohmeri as a causative pathogen. The mycology laboratory should be able to identify the yeast by prolonging its incubation on chromogenic agar and/or by using a commercial biochemical identification kit for yeasts [e.g. ID $32 \mathrm{C}$ (bioMérieux, France)]. Catheter removal is mandatory and if fluconazole is administered, the threshold to change to an antifungal regimen containing amphotericin $\mathrm{B}$, one of the newer triazoles or an echinocandin should be low.

\section{ACKNOWLEDGMENT}

The authors thank the director of UKM Medical Centre for her permission to publish this case report.

\section{REFERENCES}

1. Yang BH, Peng MY, Hou SJ, Sun JR, Lee SY, Lu JJ. Fluconazole-resistant Kodamaea ohmeri fungemia associated with cellulitis: case report and review of the literature. Int J Infect Dis 2009;13:e493-7.

2. De Barros JD, Do Nascimento SM, De Araújo FJ, Braz Rde F, Andrade VS, Theelen B, et al. Kodamaea (Pichia) ohmeri fungemia in a pediatric patient admitted in a public hospital. Med Mycol 2009;47:775-9.

3. Kriengkauykiat J, Ito JI, Dadwal SS. Epidemiology and treatment approaches in management of invasive fungal infections. Clin Epidemiol 2011;3:175-91.

4. Shang ST, Lin JC, Ho SJ, Yang YS, Chang FY, Wang NC. The emerging life-threatening 
opportunistic fungal pathogen Kodamaea ohmeri: optimal treatment and literature review. J Microbiol Immunol Infect 2010;43:200-

6.

5. Ding $\mathrm{CH}$, Wahab AA, Muttaqillah NAS, Tzar MN.

Prevalence of albicans and non-albicans

candiduria in a Malaysian medical centre. J Pak Med Assoc 2014;64:1375-9.

6. Lee JS, Shin JH, Kim MN, Jung SI, Park KH, Cho $\mathrm{D}$, et al. Kodamaea ohmeri isolates from patients in a university hospital: identification, antifungal susceptibility, and pulsed-field gel electrophoresis analysis. J Clin Microbiol 2007;45:1005-10.

7. CLSI. Reference method for broth dilution antifungal susceptibility testing of yeasts; fourth informational supplement. CLSI document M27S4. Wayne, PA: Clinical and Laboratory Standards Institute; 2012. 